\title{
Interactions between Borrelia burgdorferi and Mouse Fibroblasts
}

\author{
TOMASZ CHMIELEWSKI* and STANISŁAWA TYLEWSKA-WIERZBANOWSKA
}

\author{
Laboratory of Rickettsiae, Chlamydiae and Spirochetes, National Institute of Public Health \\ - National Institute of Hygiene, Warsaw, Poland
}

Received 18 June 2010, revised 15 July 2010, accepted 17 July 2010

\begin{abstract}
Borrelia burgdorferi spirochetes are an infectious agent of Lyme borreliosis. The aim of our studies was to investigate the fate of engulfed B. burgdorferi cells in L-929 mouse fibroblasts and to observe development of intracellular infection in vitro after 2 and $48 \mathrm{~h}$. Electron microscopic studies reveal consecutive stages of B. burgdorferi spirochetes penetration to mouse fibroblasts in vitro. It has been observed, as a first step attachment and engulfment of spirochetes followed by formation of vacuoles. After 48 hours of infection, vacuoles of fibroblastic cells have been seen full of $B$. burgdorferi bacteria and latter they have been released from infected cells to extracellular space. It can be the evidence that $B$. burgdorferi multiply intracellulary.
\end{abstract}

Ke y w ords: Borrelia burgdorferi, fibroblasts, interaction

\section{Introduction}

Borrelia burgdorferi spirochetes are an infectious agent of Lyme disease, also known as borreliosis, which is the most common tick-borne disease in the northern hemisphere. Early manifestations of infection include fever, headache, fatigue, and a characteristic skin rash called erythema migrans. Untreated, it can cause late symptoms involving tissue of the joints, heart, and nervous system (Stanek et al., 1996).

Microscopic studies indicate that the bacteria can bind to the cell surface and enter the cytoplasm directly after inducing local engulfment and fragmentation of the plasma membrane. Several reports have described interactions between $B$. burgdorferi bacteria and different host cells. It has been shown that the spirochetes can enter mammalian immune cells and other cells as well as tick tissue. This probably allows the pathogen to survive in host tissues, to infect them and to escape the host defense (Hu et al., Linder et al., 2001, Sigal, 1997, Szczepanski et al., 1990, Thomas et al., 1989). However, there is no information on the fate of B. burgdorferi spirochetes inside eukaryotic cells and the way they leave the host cells.

Various modes of bacterial entry into the host cell have been described as an essential pathogenic factors.
Legionella pneumophila uptake is completed by a process termed "bacteriopexis", followed by engulfment of the organisms with microvilli in association with intracellular cytoplasmic filaments (Oldcham et al., 1985). Another pathogen, Rickettsia prowazekii, can enter endothelial cells via induced phagocytosis and it is then released into the cell cytoplasm by disruption of the phagosomal membrane (Walker, 1984). Transmission electron microscopy has shown that viable or killed Candida organisms were attached to endothelial cells, then enveloped by cell membrane and incorporated into the endothelial cells within phagosomes (Rotrosen et al., 1985).

The ability of Borrelia burgdorferi to attach to and invade human fibroblasts was investigated by confocal and scanning electron microscopy. Scanning electron microscopy has revealed that $B$. burgdorferi are tightly attached to fibroblast monolayers after $24-48 \mathrm{~h}$. Spirochetes were observed in the perinuclear region within human fibroblasts by laser scanning confocal microscopy (Klempner et al., 1993).

The aim of our studies was to investigate the fate of engulfed B. burgdorferi cells in mouse fibroblasts and to observe the development of intracellular infection in vitro in electron and fluorescence microscopy.

* Corresponding author: T. Chmielewski, Laboratory of Rickettsiae, Chlamydiae and Spirochetes, National Institute of Public Health - National Institute of Hygiene; 24 Chocimska Street, 00-791 Warsaw;, Poland phone/fax (+48) 22 4521250; e-mail: tchmielewski@pzh.gov.pl 


\section{Experimental}

\section{Material and Methods}

Borrelia afzelii VS461 strain (ATCC 51567) was grown in BSK-H medium Complete (Sigma Aldrich, St. Louis, USA) supplemented with $6 \%$ of rabbit serum for 7 days at $35^{\circ} \mathrm{C}$ in $5 \% \mathrm{CO}_{2}$ atmosphere. The number of bacteria per $1 \mathrm{ml}$ was counted in a Thoma counting chamber.

Line L-929 (ATCC CCL-1, USA) were propagated in Eagle's minimum essential medium (MEM) with L-glutamine and $\mathrm{NaHCO}_{3}$ (Biomed, Lublin, Poland), supplemented with $4 \%$ of fetal calf serum (ATCC, USA) at $37^{\circ} \mathrm{C}$ in $5 \% \mathrm{CO}_{2}$ for 2 days. Cells were grown in shell-vials on glass coverslips inside tubes with screw caps (Sterilin, United Kingdom) until a confluent monolayer was obtained.

Bottles containing cell line monolayer were inoculated with spirochetes culture containing $10^{8}$ bacteria per $1 \mathrm{ml}$. L929 cells infected with B. burgdorferi (initial density $10^{6}$ organisms $/ \mathrm{ml}$ medium) were incubated for 2 and 48 hours at $35^{\circ} \mathrm{C}$ in $5 \% \mathrm{CO}_{2}$ atmosphere.

Infected mouse fibroblasts were fixed with acetone, washed three times with PBS and incubated 30 minutes with anti- $B$. burgdorferi human immune serum at $37^{\circ} \mathrm{C}$. Next they were washed three times with PBS followed by incubation with rabbit anti-human immunoglobulins conjugated with FITC (DAKO, Denmark). Immunofluorescence was observed in the fluorescence microscope Eclipse E 400 (NIKON, Japan) at 500X magnification.

All specimens were prepared according to standard technics (Glauert, 1975). The cultures were washed twice with PBS, fixed overnight with $2.5 \%$ glutaraldehyde. On the next day the cells were centrifuged at $750 \mathrm{~g}$ at $4^{\circ} \mathrm{C}$ and the pellet was washed with $2 \mathrm{ml}$ $0.2 \mathrm{M}$ sodium cacodylate buffer ( $\mathrm{pH} 7.4$ ) and re-centrifuged as above. After three washes, the cells were

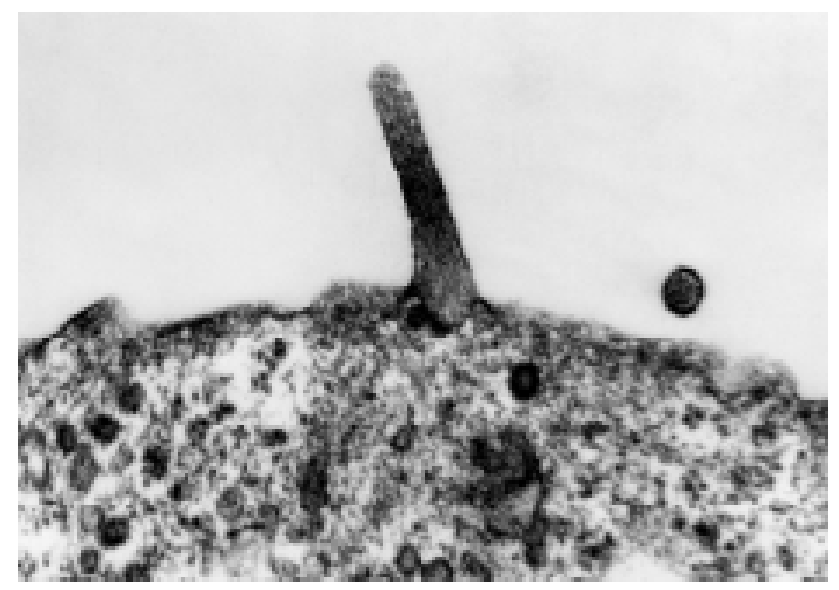

Fig. 1. Attachment of spirochetes to the cytoplasmic membrane of L-929 cell (X 30000$)$. fixed for 2 hours in $1 \% \mathrm{OsO} 4$ in $0.1 \mathrm{M}$ sodium cacodylate buffer. The cells were then washed three times in $0.1 \mathrm{M}$ sodium cacodylate buffer and stained for 30 minutes by being resuspending in $1 \%$ aqeous uranyl acetate (Roth, Karlsruhe, Germany) solution. For embedding, fibroblasts were centrifuged and then dehydrated through a wash series in methyl alcohol solutions (from $25 \%$ to $100 \%$ ) and embedded in epoxy resin and incubated overnight at $65^{\circ} \mathrm{C}$. Transverse thin sections were cut and transferred to copper mesh 300 grids (Polysciences, St. Goar, Germany), stained with lead citrate and uranyl acetate and dried. The cells were observed in a JOEL $100 \mathrm{C}$ electron microscope (Japan) at magnifications from $\times 6000$ to $\times 35000$.

\section{Results}

Various stages of B. burgdorferi spirochetes infection in L-929 fibroblasts were observed. After two hours of incubation spirochetes were seen outside of the host cells. Their position suggested that most of them were motile. The first observed interaction step was attachment of the bacteria to the surface of the fibroblasts. In fluorescence microscopy it has been seen as adhesion to the cell surface. After two hours of incubation the spirochetes were bound and entered the mammalian cells. Most of them were bound apically. This contact triggered the engulfment of the bacteria in the cytoplasmatic host membrane (Fig. 1, Fig. 2). This process initiated the cellular uptake of single bacteria into phagocytic-like vacuoles. Within two hours of incubation such bacterial cell, surrounded by host cell membrane, were seen inside the fibroblast (Fig. 3). Some bacteria entered fibroblast cells in a different way. Fibroblast pseudopods bent around single spirochetes in the intercellular spaces. The pseudopods had a characteristic hook-like form, turned back to the fibroblasts membrane and slided along the

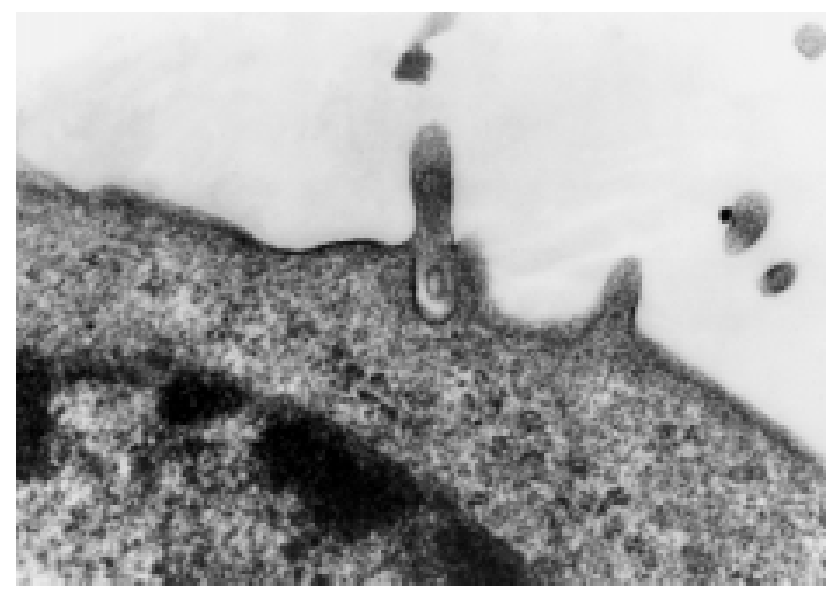

Fig. 2. Cell membrane penetration (X 30000$)$. 


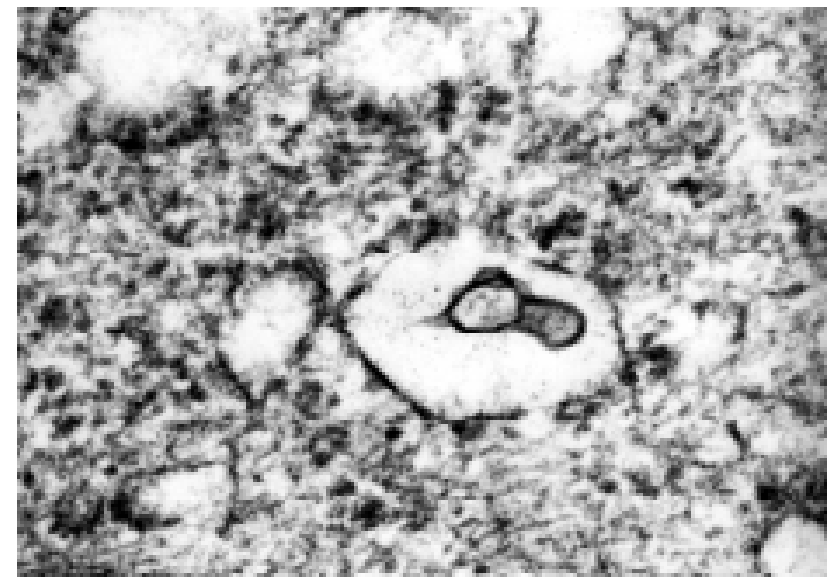

Fig. 3. Single $B$. burgdorferi bacteria in vacuole in L-929 cytoplasm (X 33000 ).

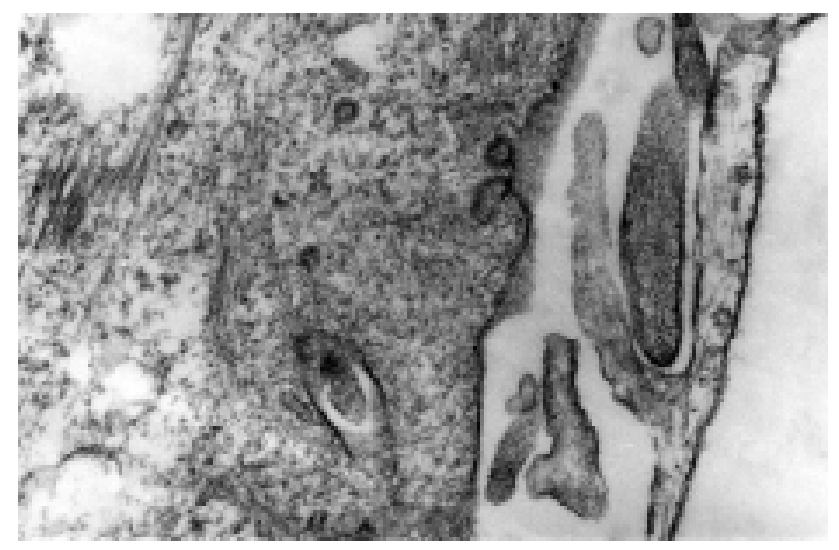

Fig. 4. Endocytosis of B. bugdorferi spirochetes (X 27000 ).

cell membrane. The observed manner of penetration resembled endocytosis however characteristic for coiling phagocytosis long appendages wrapping the spirochetes were also observed (Fig. 4).

On the second day of infection the fibroblasts were seen to be vacuolized. Many vacuoles with bacteria

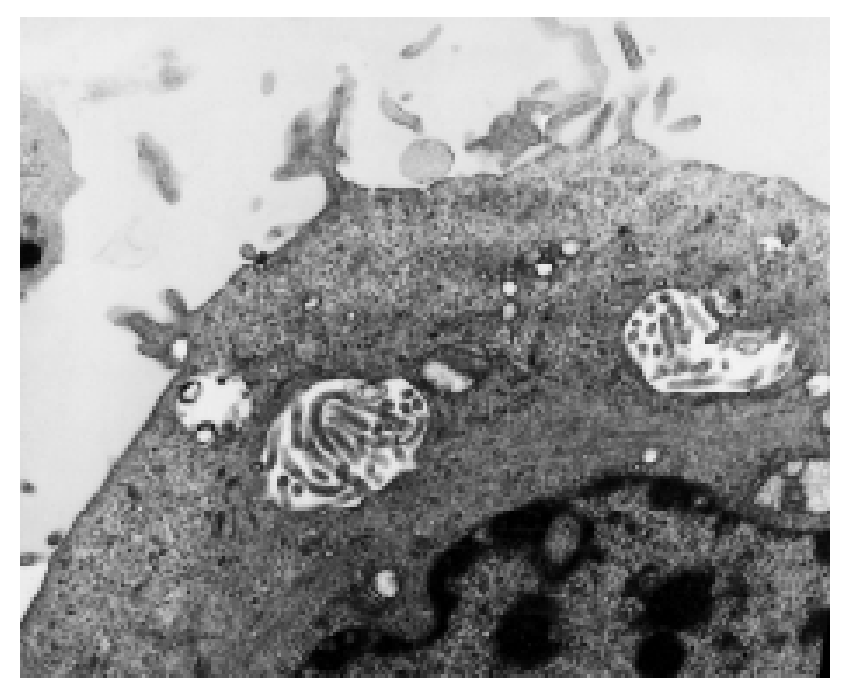

Fig. 5. Multiplication of spirochetes within vacuoles (X4 000).

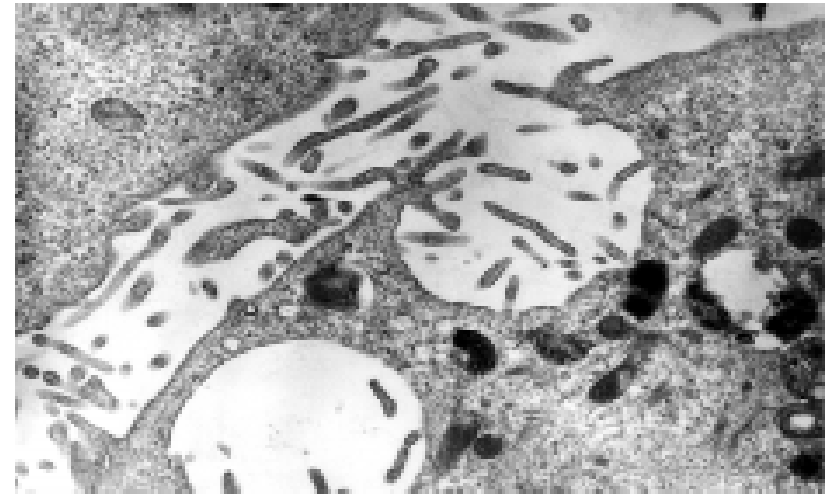

Fig. 6. Translocation of spirochetes to the intracellular space (X 6700$)$.

were located in the peripheral part of the host cell adjacent to the cell membrane. Strong immunofluorescence of vacuoles and bacteria was observed in fluorescence microscopy. The vacuoles contained 15 to 20 bacterial cells as it has been seen on a cross sections. The number of spirochetes in the host vacuoles observed after two days of infection indicated that B. burgdorferi multiplied inside the fibroblasts (Fig. 5). Some of the vacuoles were disrupted and B. burgdorferi spirochetes were released to the extracellular space (Fig. 6).

\section{Discussion}

Electron microscopic studies revealed the consecutive stages of $B$. burgdorferi spirochetes penetration into mouse fibroblasts in vitro. The first observed step was the attachment and engulfment of the spirochetes, followed by the formation of vacuoles and multiplication of bacteria inside vacuoles followed by release from the infected cells to the extracellular space. After 48 hours of infection, vacuoles of fibroblastic cells containing dozens of $B$. burgdorferi bacteria were seen. This can be taken as evidence that $B$. burgdorferi multiply intracellularly similarly to Legionella pneumophila, Coxiella burnetii and other obligatory intracellular parasites (Baca et al., 1983, Oldcham et al., 1985, Walker et al., 1984, Rotrosen et al., 1985).

Studies by Comstock et al., (1989) with electron microscopy revealed that borreliae entered the endothelial cells and suggested that the organisms penetrated the host monolayers primarily by passing through them. Attachment of spirochetes is time and temperature dependent and pretreatment with heat, immune human serum or monoclonal antibodies to OspB reduce the binding to the endothelial cells (Thomas et al., 1989). Examination of spirochete-endothelial interactions demonstrated the presence of spirochetes in the intercellular junctions between endothelial cells as well as beneath the monolayers. Scanning electron microscopy identified a mechanism of transendothelial 
migration whereby spirochetes pass between cells into the amniotic membrane at areas where subendothelium is exposed (Szczepanski et al., 1990).

Other study with Vero cells revealed that essential for the attachment process is metabolic activities of the spirochaete, not viability (ability to grow) (Hechemy et al., 1992). After entry of untreated B. burgdorferi, most of the spirochaetes were either free in the cytoplasm or tightly bound to the host membrane. In contrast, heat treated spirochaetes remained bound to host membrane in large phagosome-like vesicles (Comstock et al., 1989).

It seems that several eukaryotic cells provide $B$. burgdorferi spirochetes with a protective environment contributing to their long-term survival (Peters et al., 1997, Rittig et al., 1998, Rittig et al., 1992). B. burgdorferi have been protected in fibroblasts for at least 14 days of exposure to ceftriaxone In the absence of fibroblasts, organisms did not survive. They were not protected from ceftriaxone by glutaraldehyde-fixed fibroblasts or fibroblast lysate, suggesting that a living cell was required. The ability of the organism to survive in the presence of fibroblasts was not related to its infectivity (Georgilis et al., 1992). Mouse keratinocytes, HEp-2 cells, and Vero cells showed a similar protective effect. Doxycycline or erythromycin were more effective in killing $B$. burgdorferi when they were grown in the presence of eukaryotic cells (Brouqui et al., 1996). Our findings show that in fibroblasts could occurs process of spirochetes multiplication.

Difficulties with the isolation of $B$. burgdorferi from clinical material when cultured on artificial media also indicate that spirochetes are very fastidious bacteria, which require the presence of certain substances in the host cells for their growth. These observations allowed us to isolate several strains by inoculating cerebrospinal fluids, synovial fluids and blood of Lyme borreliosis patients into cell line culture (Chmielewski et al., 2003, Tylewska-Wierzbanowska et al., 1997).

\section{Literature}

Baca O.G. and D. Paretsky. 1983. Q fever and Coxiella burnetii: a model for host-parasite interactions. Microbiol. Rev. 47: $127-49$.

Brouqui P., S. Badiaga and D. Raoult. 1996. Eucaryotic cells protect Borrelia burgdorferi from the action of penicillin and ceftriaxone but not from the action of doxycycline and erythromycin. Antimicrob. Agents. Chemother. 40: 1552-4.

Chmielewski T., J. Fiett, M. Gniadkowski and S. TylewskaWierzbanowska. 2003. Improvement to laboratory recognition of Lyme borreliosis with the combination of culture and PCR methods. Mol. Diagn. 7: 155-162.

Comstock L.E. and D.D. Thomas. 1989. Penetration of endothelial cell monolayers by Borrelia burgdorferi. Infect. Immun. 57: $1626-1628$.

Georgilis K., M. Peacocke and M.S. Klempner. 1992. Fibroblasts protect the Lyme disease spirochete, Borrelia burgdorferi, from Ceftriaxone in vitro. J. Infect. Dis. 166: 440-444.

Glauert A.M. 1975. Practical methods in electron microscopy. In: Fixation, dehydration and embedding of biological specimens. Vol. III, part 1. North-Holland Publishing Co., Amsterdam.

Hechemy K.E., W.A. Samsonoff, H.L. Harris and M. McKee. 1992. Adherence and entry of Borrelia burgdorferi in Vero cells. J. Med. Microbiol. 36: 229-238.

Hu L.T. and M.S. Klempner. 1997. Host-pathogen interactions in the immunopathogenesis of Lyme disease. J. Clin. Immunol. 17: $354-65$.

Klempner M.S., R. Noring and R.A. Rogers. 1993. Invasion of human skin fibroblasts by the Lyme disease spirochete, Borrelia burgdorferi. J. Infect. Dis. 167: 1074-1081.

Linder S., C. Heimerl, V. Fingerle, M. Aepfelbacher and B. Wilske. 2001. Coiling phagocytosis of Borrelia burgdorferi by primary human macrophages is controlled by CDC42Hs and Rac 1 and involves recruitment of Wiskott-Aldrich syndrome protein and Arp2/3 complex. Infect. Immun. 69: 1739-46.

Oldcham L.J. and F.G. Rodgers. 1985. Adhesion, penetration and intracellular replication of Legionella pneumophila and in vitro model of pathogenesis. J. Gen. Microbiol. 131: 697-706. Peters J.D. and J.L. Benach. 1997. Borrelia burgdorferi adherence and injury to undifferentiated and differentiated neural cells in vitro. J. Infect. Dis. 176: 470-477.

Rittig M.G., J.C. Jagoda, B. Wilske, R. Murgia, M. Cinco, R. Repp, G.R. Burmester and A. Krause. 1998. Coiling phagocytosis discriminates between different spirochetes and is enhanced by phorbol myristate acetate and granulocyte-macrophage colonystimulating factor. Infect. Immun. 66: 627-35.

Rittig M.G., A. Krause, T. Häupl, U.E. Schaible, M. Modolell, M.D. Kramer and E. Lütjen-Drecoll. 1992. Coiling phagocytosis is the preferential phagocytic mechanism for Borrelia burgdorferi. Infect Immun. 60: 4205-12.

Rotrosen D., J.E. Edwards, T.R. Gibson, J.C. Moore, A.H. Cohen and I. Green. 1985. Adherence of Candida to cultered vascular endothelial cells: mechanism of attachment and endothelial cell penetration. J. Infect. Dis. 152: 1264-1273

Sigal L.H. 1997. Lyme disease: a review of aspects of its immunology and immunopathogenesis. Ann. Rev. Immunol. 15: 63-92.

Stanek G., S. O'Connel, M. Cimmino, E. Aberer, W. Kristoferitsch, M. Grandström, E. Guy and J. Gray. 1996. European Union concerted action on risk assesment in Lyme borreliosis: clinical case definitions for Lyme borreliosis. Wien. Klin. Wochenschr. 108: 741-747.

Szczepanski A., M.B. Furie, J.L. Benach, B.P. Lane and B. Fleit. 1990. Interaction between Borrelia burgdorferi and endothelium in vitro. J. Clin. Invest. 85: 1637-1647.

Thomas D.D. and L.E. Comstock. 1989. Interaction of Lyme disease spirochetes with cultured eucaryotic cells. Infect. Immun. 57: $1324-1326$

Tylewska-Wierzbanowska S. and T. Chmielewski. 1997. The isolation of Borrelia burgdorferi spirochetes from clinical material. Zbl. Bakt. 286: 363-370.

Walker T.S. 1984. Rickettsial interactions with human endothelial cells in vitro: adherence and entry. Infect. Immun. 44: 205-10 